http://dx.doi.org/10.32929/2446-8355.2019v28n3p254-267

\title{
ALTURA DE ROÇADA AFETA O CONTROLE DE CAPIM-AMARGOSO PERENIZADO
}

Ricardo Travasso Raimondi ${ }^{*}$, Jamil Constantin², Rubem Silvério de Oliveira Júnior ${ }^{2}$, Ana Karoline Silva Sanches ${ }^{3}$, Rafael Romero Mendes ${ }^{4}$

\footnotetext{
${ }^{1}$ Consultor agronômico, Agro MR, Francisco Alves, PR, Brasil.*E-mail: ricardo_raimondi@hotmail.com

${ }^{2}$ Docente, Proteção de Plantas, Universidade Estadual de Maringá, Maringá - PR.

${ }^{3}$ Mestranda, Proteção de Plantas, Universidade Estadual de Maringá, Maringá - PR.

${ }^{4}$ Doutorando, Proteção de Plantas, Universidade Estadual de Maringá, Maringá - PR.
}

Recebido: 29/10/2018; Aceito: 04/07/2019

RESUMO: Novas alternativas de controle visando à integração de métodos devem ser implementadas visando ao controle de capim-amargoso. O objetivo deste trabalho foi avaliar a influência da altura de roçada e a efetividade da aplicação de herbicidas imediatamente após a roçada no controle de capim-amargoso. Foram avaliadas seis alturas de roçada $(0,5,10,15$, 20 e $40 \mathrm{~cm})$ na presença ou ausência dos clethodim + glyphosate $\left(192+1080 \mathrm{~g} \mathrm{ha}^{-1}\right)$ aplicados imediatamente após a roçada, além de um tratamento adicional com manejo sem roçada e apenas a aplicação de herbicidas. Todos os tratamentos receberam duas aplicações sequenciais de clethodim + glyphosate, sendo a aplicação sequencial 1 com doses diferentes de clethodim nos dois experimentos (experimento $1-108 \mathrm{~g} \mathrm{ha}^{-1}$ e experimento $2-192 \mathrm{~g} \mathrm{ha}^{-1}$ ) e a aplicação sequencial 2 na dose de $192+1080 \mathrm{~g} \mathrm{ha}^{-1}$. A aplicação imediatamente após a roçada proporciona no máximo $13,8 \%$ de aumento nos níveis de controle ao final do sistema de manejo. Quanto menor a altura de roçada, maior é o controle de capim-amargoso, sendo que as alturas iguais ou menores a $20 \mathrm{~cm}$ resultam em controle superiores ao tratamento apenas com herbicidas. A roçada associada a um herbicida pode constituir em importante ferramenta para o manejo de capim-amargoso.

Palavras-chave: Digitaria insularis. Controle mecânico. Clethodim. Rizoma. Manejo integrado.

\section{MOWING HEIGHT AFFECTS CLUMPED SOURGRASS CONTROL}

ABSTRACT: New alternatives with integrated methods must be implemented for sourgrass control. The aim of this work was to evaluate the influence of mowing height and to evaluate the viability of herbicide application immediately after mowing in the control of sourgrass. Six mowing heights $(0,5,10,15,20$ and $40 \mathrm{~cm})$ were evaluated in the presence or absence of clethodim + glyphosate $\left(192+1080 \mathrm{~g} \mathrm{ha}^{-1}\right)$ application immediately after mowing, plus an additional treatment with only herbicide applications. All treatments received two sequential applications of clethodim + glyphosate. The sequential application 1 was applied in different doses of clethodim between two experiments (experiment $1-108 \mathrm{~g} \mathrm{ha}^{-1}$ and experiment 2 $192 \mathrm{~g} \mathrm{ha}^{-1}$ ) and sequential application 2 in dose of $192+1080 \mathrm{~g} \mathrm{ha}^{-1}$. Application immediately after mowing provides maximum of $13,8 \%$ in control levels at the end of the management 
system. The lowers mowing heights provide greater sourgrass control, and mowing similar or smaller than $20 \mathrm{~cm}$ result in superior control than treatment with only herbicides. The mowing can be an important tool for sourgrass management when associated with herbicide applicatinos.

Key words: Digitaria insularis. Mechanical control. Clethodim. Rhizome. Integrated management.

\section{INTRODUÇÃO}

O capim-amargoso (Digitaria insularis) é uma planta daninha problemática no Brasil devido a algumas características de agressividade como ciclo perene, reprodução vegetativa por rizomas, elevada taxa de produção, dispersão e germinação de sementes (GEMELLI et al., 2012; LORENZI, 2014; MARTINS et al., 2017). Esta espécie assumiu maior importância após a seleção de populações resistentes ao glyphosate em locais onde este herbicida era utilizado com frequência, como pomares (CARVALHO et al., 2011) e culturas tolerantes (ADEGAS et al., 2010). Atualmente, populações de capim-amargoso resistentes ao glyphosate são encontradas em mais de dez estados do Brasil (LOPEZ OVEJERO et al., 2017) causando prejuízos especialmente nas regiões tropicais em culturas anuais como soja e milho.

O controle de capim-amargoso é realizado basicamente com o uso de herbicidas. Existem algumas opções de mecanismos de ação para o controle desta espécie em pósemergência inicial (até três perfilhos), como por exemplo, inibidores da glutamina-sintetase, inibidores do fotossistema I e inibidores da acetolactato-sintate (SILVA et al., 2017). Contudo, em plantas adultas, após a formação dos rizomas que coincide aproximadamente com 45 dias após a emergência, apenas os inibidores da acetil-coenzima-A (ACCase) têm sido eficientes para o controle de populações resistentes ao glyphosate (MELO et al., 2012; LICORINI et al., 2015). Mesmo assim, em algumas situações, são necessárias duas ou mais aplicações sequenciais para obter bons resultados (GEMELLI et al., 2013; ZOBIOLE et al., 2016).

Neste contexto, considera-se preocupante a possibilidade de seleção de populações de capim-amargoso com resistência múltipla a glyphosate e inibidores da ACCase. Outras alternativas ao controle químico vêm sendo empregadas em áreas de produção de grãos do Brasil, sendo a principal o revolvimento do solo com práticas de aração e gradagem. Entretanto, tais métodos de controle trazem consigo implicações negativas para o manejo do solo como perda de matéria orgânica, compactação e exposição a processos erosivos (LISBOA et al., 2012). Por conseguinte, alguns produtores estão escolhendo a roçada das plantas de capim-amargoso como método de controle mecânico.

$\mathrm{Na}$ operação de roçada é realizado o corte da parte aérea das plantas rente ao solo, o que é uma prática muito comum em cultivos perenes e o controle de plantas daninhas é realizado nas entrelinhas das culturas (SILVA et al., 2011). Considerando plantas adultas de capimamargoso, o corte da parte aérea não é suficiente para a eliminação das plantas, uma vez que a produção dos rizomas abaixo do nível do solo permite a emissão de novos perfilhos. Portanto, 
espera-se que a roçada represente uma das ferramentas dentro de um sistema de manejo de capim-amargoso, envolvendo programas integrados de controle mecânico e químico.

Existem possibilidades de diferentes alturas de roçada influenciarem o controle de capim-amargoso quando associados ao controle químico. Em pastagens, é comum o corte das plantas seguido de aplicação de herbicidas diretamente no toco em espécies arbóreas (MENDES et al., 2016). Apesar de não avaliado em gramíneas, pode haver absorção e translocação dos herbicidas aplicados logo após a roçada, de modo a intoxicar as estruturas vegetativas abaixo do solo para evitar novos rebrotes. Os objetivos deste trabalho, portanto, foram (a) avaliar a relação entre a altura de roçada - integrada ao controle químico - e a eficiência de controle de capim-amargoso; e (b) avaliar a viabilidade de se realizar uma aplicação de herbicidas logo após a roçada no controle de capim-amargoso perenizado.

\section{MATERIAL E MÉTODOS}

Foram conduzidos simultaneamente dois experimentos a campo no período de 15/09 a 30/11 de 2016, no município de Mandaguaçú - PR (coordenadas 23 14'35' S; 5200'24" $\mathrm{O})$. Os experimentos foram instalados lado a lado e o solo da área experimental apresentava pH em H2O de 6,0; carbono orgânico a 21,0 mg dm-3; 11,9\% de areia, 16,1\% de silte e 63\% de argila. A área experimental apresentava infestação de capim-amargoso adulto (com continua produção de sementes) e resistente ao glyphosate (3-4 plantas $\mathrm{m}^{2}$ ) e estava em pousio por um mês após a colheita de milho segunda safra. Os dados climáticos durante o período experimental apresentam-se na Figura 1.

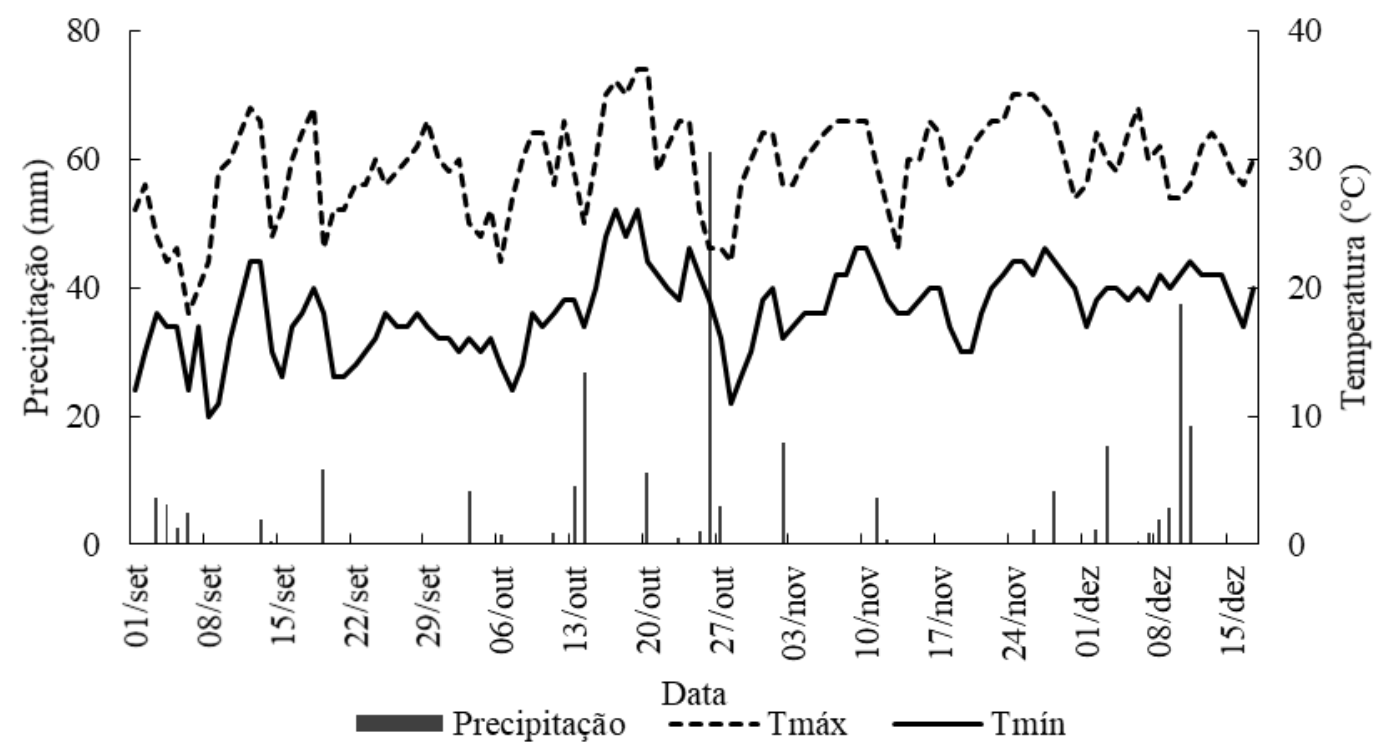

Figura 1. Dados de temperatura (T) máxima e mínima e precipitação durante o período experimental. INMET, Maringá (PR), 2018. Data of maximum and minimum temperature (T) and precipitation during the experimental period. INMET, Maringá (PR), 2018.

Fonte: INMET (2018).

O delineamento experimental utilizado foi o de blocos ao acaso, com quatro repetições. Os tratamentos compuseram em parcelas subdivididas $(6 \times 2)+1$, em que o primeiro fator, disposto nas parcelas, foi representado por seis alturas de roçada do capim-amargoso $(0,5,10$, 
15,20 e $40 \mathrm{~cm}$ ) e o segundo fator, disposto nas subparcelas, foi composto pela ausência ou presença de aplicação de clethodim a 192 g ha-1 imediatamente após a roçada. O tratamento adicional foi representado por um método de controle sem roçada, apenas com a aplicação de clethodim a $192 \mathrm{~g}$ ha-1, considerado como padrão. As parcelas foram dimensionadas com 16 $\mathrm{m}^{2}(2 \times 8 \mathrm{~m})$ e as subparcelas com $8 \mathrm{~m}^{2}(2 \mathrm{x} 4 \mathrm{~m})$.

Em todos os tratamentos de ambos os experimentos foram realizadas duas aplicações sequenciais, sendo $108 \mathrm{~g}$ ha-1 / $108 \mathrm{~g}$ ha-1 de clethodim (Experimento 1) e 192 / $108 \mathrm{~g}$ ha-1 de clethodim (Experimento 2). Portanto, o que diferenciou os dois experimentos foi a dose da primeira aplicação sequencial. Em todas as aplicações foram adicionados glyphosate $(1080 \mathrm{~g}$ e.a. ha-1) e óleo mineral ( $0,05 \% \mathrm{v} v-1)$. Estas aplicações foram realizadas quando o rebrote médio do capim-amargoso nos experimentos apresentava altura de $15 \mathrm{~cm}$ (RAIMONDI, 2018). Para os dois experimentos, a aplicação sequencial 1 (Seq.1) foi realizada 25 dias após a roçada (DAR) e a aplicação sequencial 2 (Seq.2) aos 45 DAR (Tabela 1).

Tabela 1. Datas das operações e avaliações realizadas nos experimentos. Maringá, PR, 2018. Dates of operations and evaluations performed in the experiments. Maringá, PR, 2018.

\begin{tabular}{|c|c|c|}
\hline$\overline{\text { Data (2016) }}$ & Operação & Avaliação \\
\hline $15 /$ set & Roçada ou roçada+aplicação ${ }^{1 /}$ & Área foliar e massa seca de folhas \\
\hline $30 /$ set & Aplicação Seq. $1^{2 /}$ & Controle 15 DAR \\
\hline $10 /$ out & - & Controle 25 DAR e 10 DAAS-1 \\
\hline 20/out & - & Controle 35 DAR e 20 DAAS-1 \\
\hline 30/out & Aplicação Seq.2 & Controle 45 DAR e 30 DAAS-1 \\
\hline $15 /$ nov & - & Controle 60 DAR, 45 DAAS-1 e 15 DAAS-2 \\
\hline $25 /$ nov & - & Controle 70 DAR, 55 DAAS-1 e 25 DAAS-2 \\
\hline \multicolumn{3}{|c|}{ 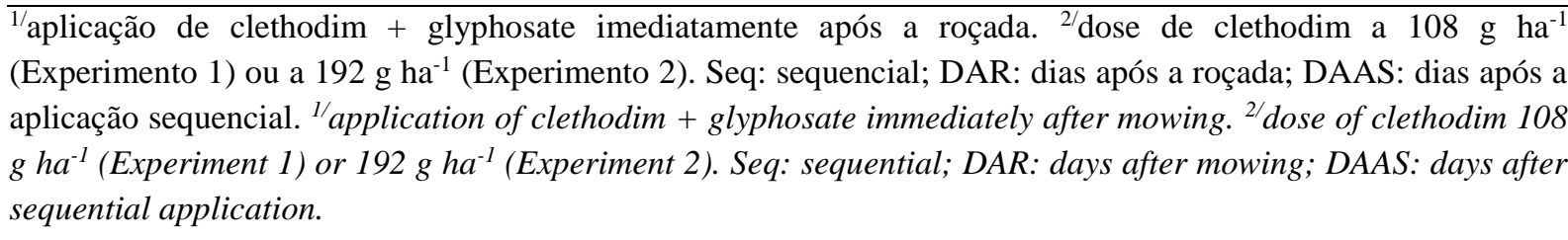 } \\
\hline
\end{tabular}

A roçada do capim-amargoso foi realizada com auxílio de roçadora portátil Sthil (FS220) a combustão, com uma lâmina de aço com três pontas. A altura de roçada foi considerada medindo-se a altura do solo até a inserção da primeira folha. Para as aplicações, foi utilizado um pulverizador costal à base de $\mathrm{CO}_{2}$ sob pressão constante, equipado com uma barra com quatro pontas do tipo leque ST 0.15, espaçadas $0,5 \mathrm{~m}$ entre si. A pressão de trabalho foi de 28,44719 Psi e a velocidade foi de $1,0 \mathrm{~m} \mathrm{~s}^{-1}$, o que proporcionou volume de aplicação de $150 \mathrm{~L} \mathrm{ha}^{-1}$.

Logo após a operação de roçada, a parte aérea de uma planta de cada subparcela em cada experimento foi cortada para a mensuração da área foliar das folhas remanescentes no medidor LI-COR ${ }^{\circledR}$, modelo LI 3100.

O efeito dos tratamentos foi avaliado, observando-se o controle de capim-amargoso, considerando a escala de $0-100 \%$, em que 0\% significava ausência de sintomas e $100 \%$ a morte da planta (SBCPD, 1995). As datas das avaliações encontram-se na Tabela 1. 
Os dados de controle foram submetidos à análise de variância, ao nível de $5 \%$ de probabilidade. Para o fator altura de roçadas, modelos lineares foram ajustados aos dados e o fator ausência ou presença de aplicação após a roçada foi comparado pelo teste $\mathrm{F}(5 \%)$. $\mathrm{O}$ tratamento padrão foi comparado com os demais pela decomposição da soma de quadrado dos tratamentos em contrastes ortogonais, ao nível de 5\% de probabilidade. O programa estatístico utilizado foi o SISVAR 12 e o software Excel para estimar a soma de quadrados da testemunha adicional e para as correções dos valores de F calculados.

\section{RESULTADOS E DISCUSSÃO}

Não houve interação entre os fatores em nenhum dos experimentos assim como nas avaliações. Optou-se por fazer o desdobramento do primeiro fator (altura de roçada) dentro de cada nível do segundo fator (ausência ou presença de aplicação imediatamente após a roçada), para ambos os experimentos. Em todas as avaliações constatou-se aumentos nos níveis de controle de capim amargoso na presença de aplicação imediatamente após a roçada, sendo este aumento de no máximo $13,8 \%$ (Figura 2). Não foi observado controle acima de $85 \%$ na média de todas as alturas de roçada até os 45 DAR para ambos os experimentos. Apenas após a Seq.2 (60 DAR em diante) observou-se níveis de controle acima de 90\%. Na avaliação final (70 DAR), o aumento no controle em função da aplicação imediatamente após a roçada foi de $4,2 \%$ e $3,4 \%$ para o experimento 1 e 2 , respectivamente (Figura 2).

Para o controle de plantas daninhas arbóreas em pastagens, a aplicação de herbicida sistêmicos no caule imediatamente após o corte da parte aérea proporciona a translocação dos herbicidas pelo floema (MENDES et al., 2016). Todavia, a aplicação nos restos culturais do capim-amargoso após a roçada não suprimiu o rebrote das plantas, provavelmente pela limitada absorção e translocação dos herbicidas pelos colmos e raízes. Em gramíneas, além da limitada área para absorção pelos colmos cortados, o sistema vascular é menos organizado e pode dificultar a translocação para as raízes. O acréscimo no controle de capim-amargoso pode estar relacionado com a intoxicação causada nas maiores alturas de roçada $(20$ e $40 \mathrm{~cm})$ em que houve área foliar remanescente suficiente para a absorção dos herbicidas aplicados imediatamente após o corte (Figura 3). 

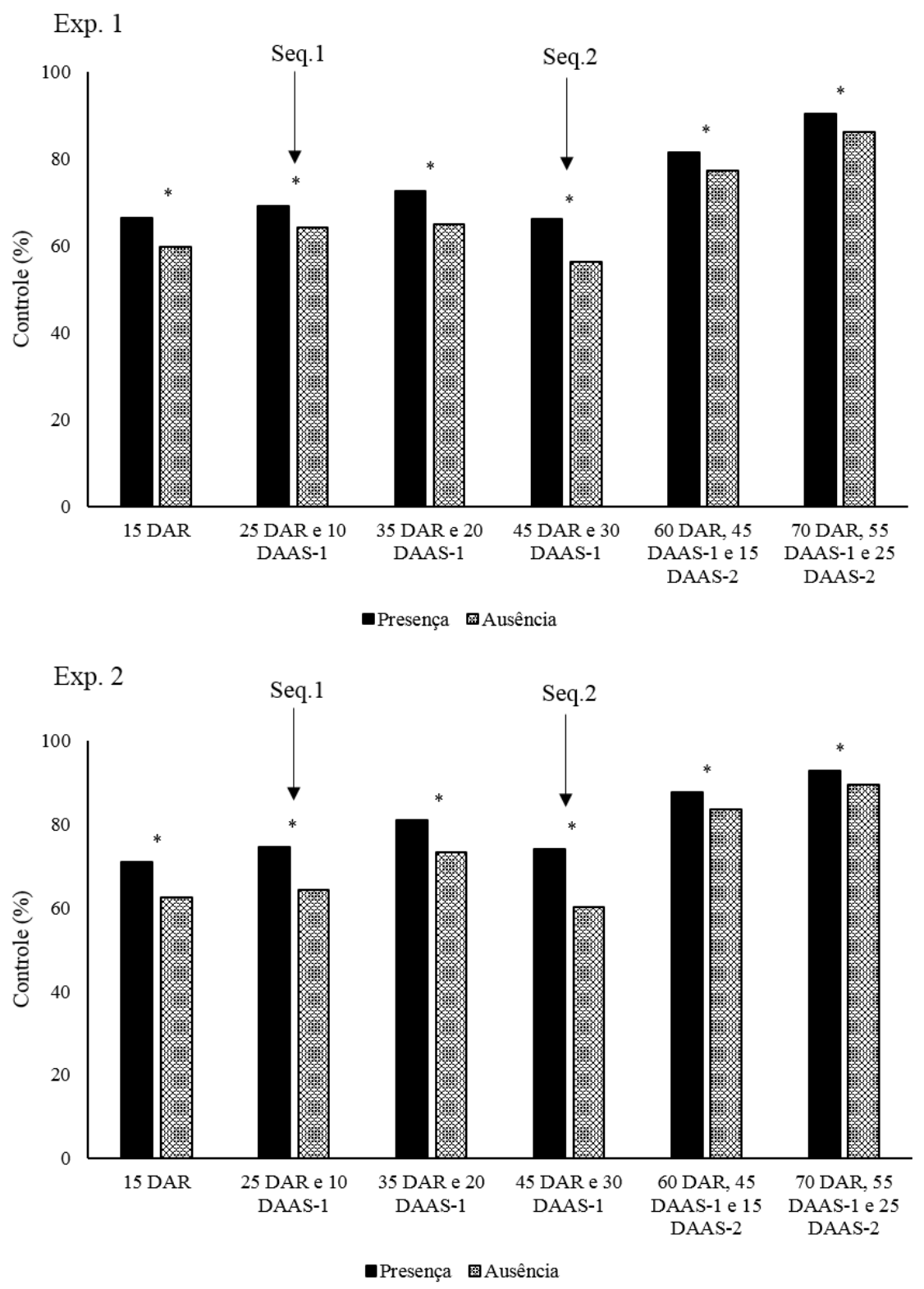

DAR: dias após a roçada; DAAS: dias após a aplicação sequencial. *significativo pelo teste $\mathrm{F}$ a $5 \%$ de probabilidade. Seq. 1: aplicação sequencial 1 - chethodim + glyphosate $(108+1080 \mathrm{~g}$ ha-1 para o experimento 1 e $192+1080 \mathrm{~g}$ ha-1 para o experimento 2). Seq.2: aplicação sequencial 2 - clethodim + glyphosate $(192+1080$ g ha-1). DAR: days after mowing; DAAS: days after sequential application. *significant by the $F$ test, at 5\% probability. Seq.1: sequential application 1-chethodim + glyphosate $(108+1080 \mathrm{~g}$ ha-1 for experiment 1 and $192+1080 \mathrm{~g}$ ha-1 for experiment 2). Seq. 2: sequential application 2-clethodim + glyphosate $(192+1080 \mathrm{~g}$ ha1).

Figura 2. Controle de capim-amargoso na presença ou ausência de aplicação de clethodim + glyphosate $\left(192+1080 \mathrm{~g} \mathrm{ha}^{-1}\right)$ imediatamente após a roçada (média de seis alturas de roçada: $0,5,10,15,20$ e $40 \mathrm{~cm})$. Maringá, PR, 2018. Sourgrass control in the presence or absence of application of clethodim + glyphosate $\left(192+1080 \mathrm{~g} \mathrm{ha}^{-1}\right)$ immediately after mowing (mean of six mowing heights: 0, 5, 10,15,20 and $40 \mathrm{~cm}$ ). Maringá, PR, 2018. 


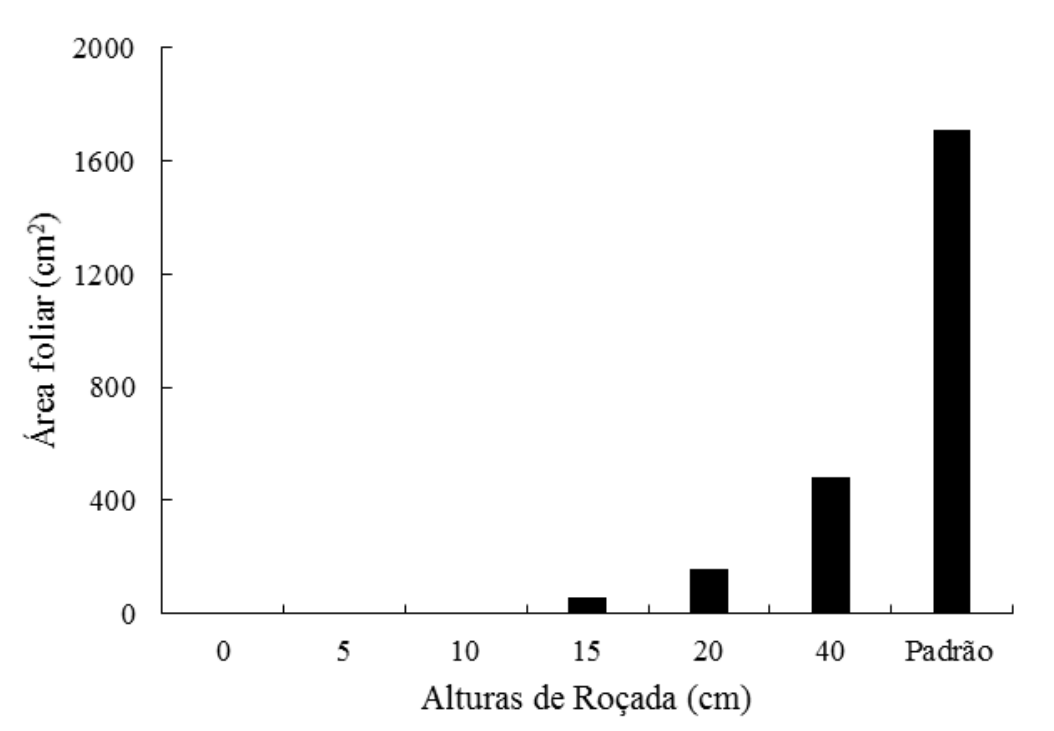

Padrão: tratamento sem roçada, apenas com aplicação de herbicidas. Check: treatment no mowing, only with herbicide application.

Figura 3. Área foliar remanescente após a roçada de capim-amargoso a diferentes alturas (média de dois experimentos). Remaining leaf area after sourgrass mowing at different heights (mean of two experiments).

Os modelos lineares ajustados nos dois experimentos indicaram que quanto maior a altura de roçada, menor os níveis de controle de capim-amargoso (Figuras 4 e 5). Os coeficientes lineares obtidos no experimento 1 , os quais representam o máximo controle estimado pelos modelos, foram superiores a $85 \%$ somente após as duas aplicações sequencias (60 DAR). Isso indica que são necessárias pelo menos duas aplicações posteriores de herbicidas para alcançar bons resultados, mesmo realizando a roçada ao nível do solo $(0 \mathrm{~cm})$ (Figura 4). O presente trabalho teve resultados semelhantes ao trabalho de Correia et al., (2015), onde, resultados superiores de controle foram observados quando ocorreu aplicação sequencial em relação às aplicações únicas de inibidores da ACCase + glyphosate. Da mesma forma, glyphosate + inibidores da ACCase seguidos de uma aplicação de inibidores da ACCase isoladamente apresentam resultados superiores em comparação com aplicações únicas em plantas adultas de capim-amargoso (GILO et al., 2016). 

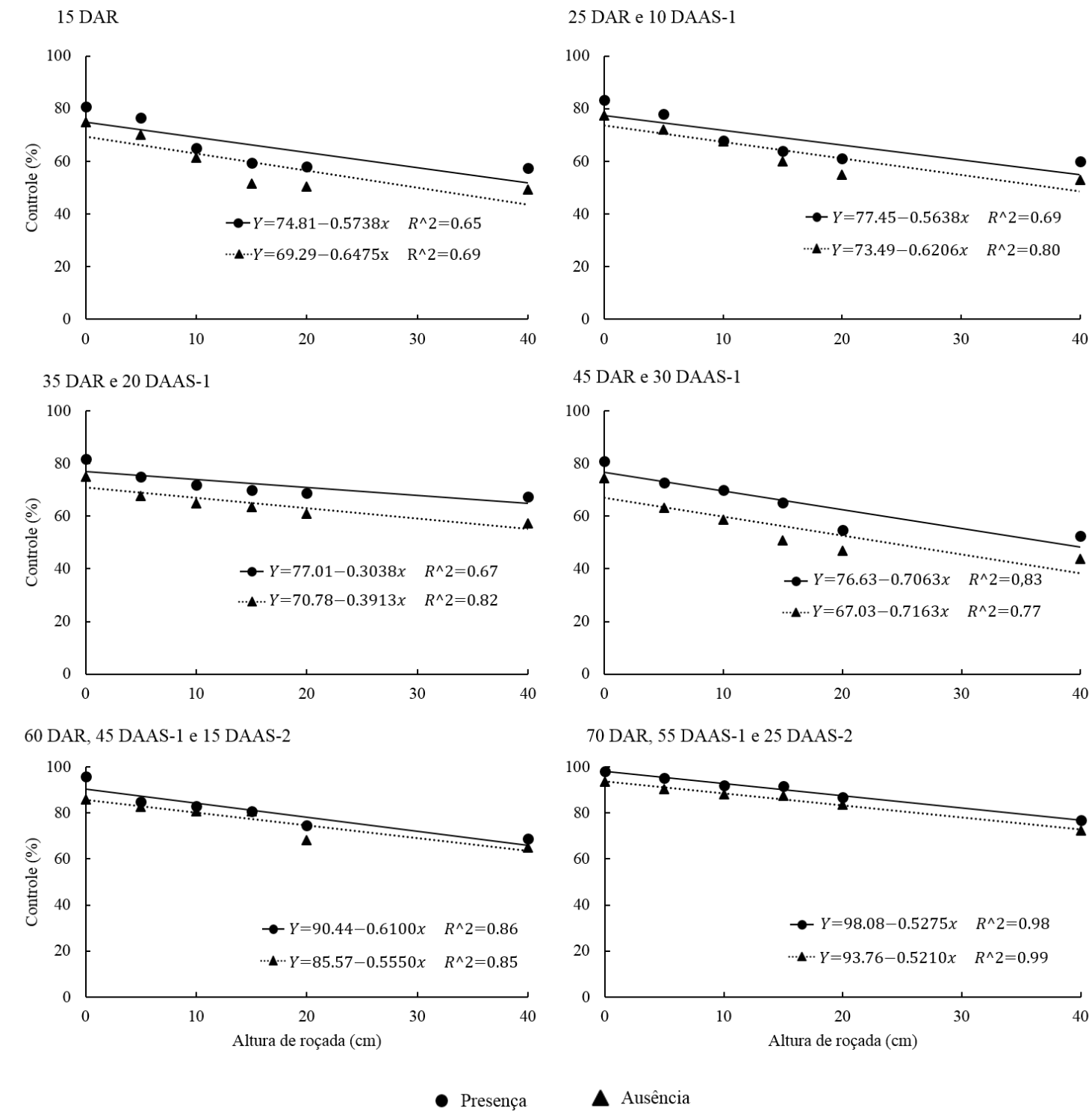

70 DAR, 55 DAAS-1 e 25 DAAS-2

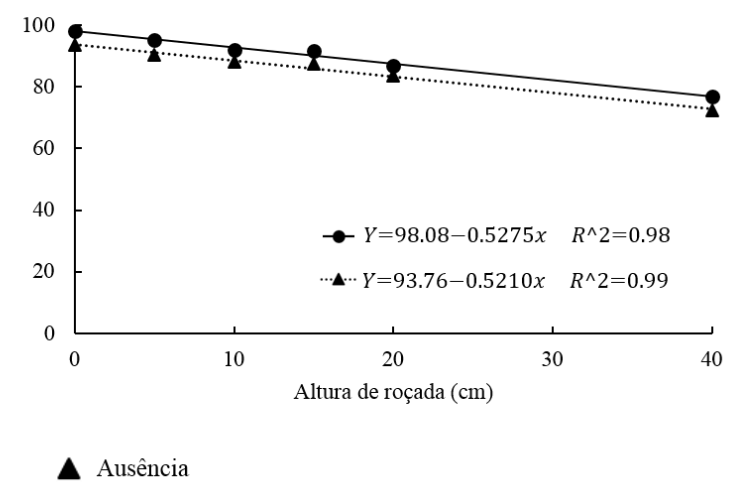

DAR: dias após a roçada; DAAS: dias após a aplicação sequencial. DAM: days after mowing; DASA: days after sequential application.

Figura 4. Controle de capim-amargoso em função da altura de roçada na presença ou ausência de aplicação de clethodim + glyphosate $\left(192+1080 \mathrm{~g} \mathrm{ha}^{-1}\right)$ imediatamente após a roçada. Experimento 1. Aplicação Seq.1 - 25 dias após a roçada com clethodim + glyphosate $(108+1080 \mathrm{~g}$ ha-1). Aplicação Seq. 2 - 45 dias após a roçada com clethodim + glyphosate $(108+1080 \mathrm{~g}$ ha-1). Sourgrass control as a function of the mowing height in the presence or absence of application of clethodim + glyphosate $\left(192+1080 \mathrm{~g} \mathrm{ha}^{-1}\right)$ immediately after mowing. Experiment 1. Application Seq.1 - 25 days after mowing with clethodim + glyphosate $\left(108+1080 \mathrm{~g} \mathrm{ha}^{-1}\right)$. Application Seq.2 - 45 days after mowing with clethodim + glyphosate $\left(108+1080 \mathrm{~g} \mathrm{ha}^{-1}\right)$.

Com relação ao experimento 2, também só se obteve níveis de controle acima de $90 \%$ com a roçada a $0 \mathrm{~cm}$ após a Seq.2 (60 DAR em diante), tanto na ausência quanto na presença de aplicações imediatamente após a roçada (Figura 5). Aparentemente, não há incremento nos resultados finais quando a dose de clethodim é maior - utilizada no experimento 2 - na aplicação sobre o primeiro rebrote do capim-amargoso após a roçada (Seq.1). 

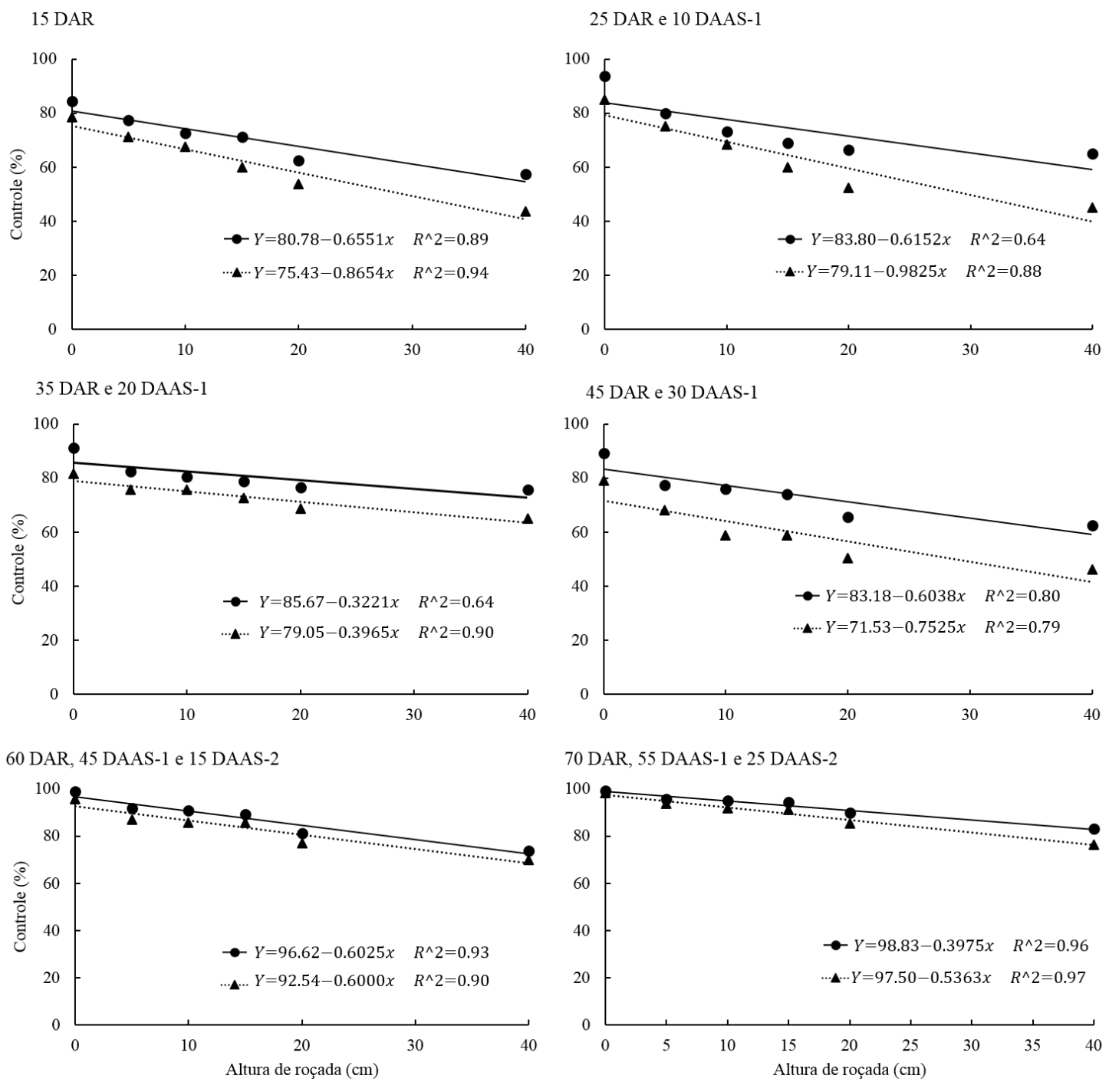

- Presença

A Ausência

DAR: dias após a roçada; DAAS: dias após a aplicação sequencial. DAM: days after mowing; DASA: days after sequential application.

Figura 5. Controle de capim-amargoso em função da altura de roçada na presença ou ausência de aplicação de clethodim + glyphosate $\left(192+1080 \mathrm{~g} \mathrm{ha}^{-1}\right)$ imediatamente após a roçada. Experimento 2. Aplicação Seq. 1 - 25 dias após a roçada com clethodim + glyphosate $\left(192+1080 \mathrm{~g} \mathrm{ha}^{-1}\right)$. Aplicação Seq. $2-45$ dias após a roçada com clethodim + glyphosate $\left(108+1080 \mathrm{~g} \mathrm{ha}^{-1}\right)$. Sourgrass control as a function of mowing height in the presence or absence of application of clethodim + glyphosate $\left(192+1080 \mathrm{~g} \mathrm{ha}^{-1}\right)$ immediately after mowing. Experiment 2. Application Seq.1 - 25 days after mowing with clethodim + glyphosate $\left(192+1080 \mathrm{~g} \mathrm{ha}^{-1}\right)$. Application Seq. $2-45$ days after mowing with clethodim + glyphosate $\left(108+1080 \mathrm{~g} \mathrm{ha}^{-1}\right)$.

Ao comparar os tratamentos de roçada com o tratamento padrão (apenas aplicações de herbicidas) no experimento 1 (Tabela 2) observou-se que os níveis de controle são maiores no início do manejo (15 DAR). As alturas de roçada de $0,5,10,15$ e $20 \mathrm{~cm}$ apresentaram resultados de controle semelhantes ou melhores em relação ao tratamento padrão na avaliação 
realizada ao final do experimento (70 DAR). A roçada na altura de $40 \mathrm{~cm}$ não constitui um tratamento recomendado por apresentar níveis de controle inferiores.

Tabela 2. Controle de capim-amargoso em diferentes alturas de roçada na ausência e presença da aplicação de clethodim + glyphosate $\left(192+1080 \mathrm{~g} \mathrm{ha}^{-1}\right)$ imediatamente após a roçada e comparação com o controle exclusivamente químico. Experimento 1. Maringá, PR, 2018. Sourgrass control at different mowing heights in the absence and presence of the application of clethodim + glyphosate $\left(192+1080 \mathrm{~g} \mathrm{ha}^{-1}\right)$ immediately after mowing and comparison with the exclusively chemical control. Experiment 1. Maringá, PR, 2018.

\begin{tabular}{ccccccc}
\multirow{2}{*}{$\begin{array}{c}\text { Altura de } \\
\text { roçada }(\mathrm{cm})\end{array}$} & \multicolumn{2}{c}{ 15 DAR } & \multicolumn{2}{c}{ 35 DAR e 20 DAAS-1 } & \multicolumn{2}{c}{$\begin{array}{c}\text { 70 DAR, 55 DAAS-1 e } \\
\text { 25 DAAS-2 }\end{array}$} \\
\cline { 2 - 7 } & Presença & Ausência & Presença & Ausência & Presença & Ausência \\
\hline 0 & $80,8^{(+)}$ & $75,0^{(+)}$ & $81,8^{(+)}$ & 75,0 & $98,3^{(+)}$ & $93,8^{(+)}$ \\
5 & $76,5^{(+)}$ & $70,0^{(+)}$ & 75,0 & 67,8 & $95,3^{(+)}$ & $90,3^{(+)}$ \\
10 & $65,0^{(+)}$ & $61,2^{(+)}$ & 71,8 & 65,0 & $92,0^{(+)}$ & 88,3 \\
15 & $59,5^{(+)}$ & $51,5^{(+)}$ & 70,0 & $63,5^{(-)}$ & $91,8^{(+)}$ & 87,5 \\
20 & $58,0^{(+)}$ & 50,5 & 68,8 & $61,0^{(-)}$ & 86,8 & 83,5 \\
40 & $57,5^{(+)}$ & 49,3 & 67,5 & $57,3^{(-)}$ & $77,0^{(-)}$ & $72,5^{(-)}$ \\
\hline
\end{tabular}

Padrão $^{1 /} \quad 45,0 \quad 70,0 \quad 83,3$

DAR: dias após a roçada; DAAS: dias após a aplicação sequencial. (+) indica que o tratamento foi superior ao padrão e (-) indica que o tratamento foi inferior ao padrão, pela estimativa de contrastes ortogonais a 5\% de probabilidade. ${ }^{1 / a p e n a s ~ h e r b i c i d a ~(c h e t h o d i m ~+~ g l y p h o s a t e ~}-192 \mathrm{e} 1080 \mathrm{~g} \mathrm{ha}^{-1}$ ). Todos os tratamentos receberam duas aplicações sequenciais: aplicação Seq. $1-25$ dias após a roçada com clethodim + glyphosate $(108+1080 \mathrm{~g}$ $\left.\mathrm{ha}^{-1}\right)$. Aplicação Seq. $2-45$ dias após a roçada com clethodim + glyphosate $\left(108+1080 \mathrm{~g} \mathrm{ha}^{-1}\right)$. DAR: days after mowing; DAAS: days after sequential application. $(+)$ indicates that the treatment was superior to the standard and (-) indicates that the treatment was lower than the standard, by orthogonal contrast estimation, at 5\% probability. "herbicide only (chethodim + glyphosate -192 and $1080 \mathrm{~g} \mathrm{ha}^{-1}$ ). All treatments received two sequential applications: application Seq. $1-25$ days after mowing with clethodim + glyphosate $(108+1080 \mathrm{~g}$ $\left.h \mathrm{~h}^{-1}\right)$. Application Seq. 2 - 45 days after mowing with clethodim + glyphosate $\left(108+1080 \mathrm{~g} \mathrm{ha^{-1 }}\right)$.

Resultados semelhantes foram observados na primeira avaliação (15 DAR) realizada no experimento 2. Após as aplicações sequenciais, os tratamentos com roçada $\leq 15 \mathrm{~cm}$ foram considerados vantajosos em relação ao tratamento padrão. Para as maiores alturas de roçada $(20$ e $40 \mathrm{~cm}$ ) os resultados foram semelhantes ao manejo apenas com herbicidas (Tabela 3).

Os experimentos demonstraram que apenas uma aplicação de clethoim + glyphosate imediatamente após a roçada proporciona aumento nos níveis de controle de capim amargoso, entretanto, este aumento é insatisfatório ao final de um sistema de manejo.

A roçada pode substituir uma aplicação de herbicida (Tabelas 2 e 3), contudo, apenas a roçada não é suficiente para o controle de capim-amargoso, devido à capacidade da emissão de novos rebrotes (Raimondi, 2018). Assim como no presente trabalho, Dantas et al. (2015) constataram que o manejo químico com glyphosate + clethodim dez dias após a roçada proporciona níveis de controle acima de 90\%. Portanto, à medida que ocorrem os fluxos de parte aérea (rebrote de $15 \mathrm{~cm}$ ), são necessárias novas intervenções de controle, integrando o método mecânico e químico. 
Tabela 3. Controle de capim-amargoso em diferentes alturas de roçada na ausência e presença da aplicação de clethodim + glyphosate $\left(192+1080 \mathrm{~g} \mathrm{ha}^{-1}\right)$ imediatamente após a roçada e comparação com o controle exclusivamente químico. Experimento 2. Maringá, PR, 2018. Sourgrass control at different brush heights in the absence and presence of clethodim + glyphosate $\left(192+1080 \mathrm{~g} \mathrm{ha}^{-1}\right)$ immediately after mowing and comparison with the exclusively chemical control. Experiment 2. Maringá, PR, 2018.

\begin{tabular}{|c|c|c|c|c|c|c|}
\hline \multirow{2}{*}{$\begin{array}{l}\text { Altura de } \\
\text { roçada }(\mathrm{cm})\end{array}$} & \multicolumn{2}{|c|}{15 DAR } & \multicolumn{2}{|c|}{35 DAR e 20 DAAS-1 } & \multicolumn{2}{|c|}{$\begin{array}{c}70 \text { DAR, } 55 \text { DAAS-1 e } \\
25 \text { DAAS-2 }\end{array}$} \\
\hline & Presença & Ausência & Presença & Ausência & Presença & Ausência \\
\hline 0 & $84,5^{(+)}$ & $78,5^{(+)}$ & $91,3^{(+)}$ & $81,5^{(+)}$ & $99,3^{(+)}$ & $98,3^{(+)}$ \\
\hline 5 & $77,5^{(+)}$ & $71,3^{(+)}$ & $82,5^{(+)}$ & 75,8 & $95,8^{(+)}$ & $93,8^{(+)}$ \\
\hline 10 & $72,5^{(+)}$ & $67,5^{(+)}$ & 80,5 & 75,8 & $95,0^{(+)}$ & $91,8^{(+)}$ \\
\hline 15 & $71,3^{(+)}$ & $60,0^{(+)}$ & 78,8 & 72,5 & $94,5^{(+)}$ & $91,3^{(+)}$ \\
\hline 20 & $62,5^{(+)}$ & $53,8^{(+)}$ & 76,5 & $68,8^{(-)}$ & 89,8 & 85,5 \\
\hline 40 & $57,5^{(+)}$ & 43,8 & 75,8 & $65,0^{(-)}$ & 83,0 & 76,3 \\
\hline Padrão $^{1 /}$ & \multicolumn{2}{|c|}{46,5} & \multicolumn{2}{|c|}{74,2} & \multicolumn{2}{|c|}{85,0} \\
\hline
\end{tabular}

(+) indica que o tratamento foi superior ao padrão e (-) indica que o tratamento foi inferior ao padrão, pela estimativa de contrastes ortogonais, à $5 \%$ de probabilidade. ${ }^{1 /}$ apenas herbicida (chethodim + glyphosate $-192 \mathrm{e}$ $\left.1080 \mathrm{~g} \mathrm{ha}^{-1}\right)$. Todos os tratamentos receberam duas aplicações sequenciais: aplicação Seq.1 -25 dias após a roçada com clethodim + glyphosate $\left(192+1080 \mathrm{~g} \mathrm{ha}^{-1}\right)$. Aplicação Seq. $2-45$ dias após a roçada com clethodim + glyphosate $\left(108+1080 \mathrm{~g} \mathrm{ha}^{-1}\right)$. DAR: dias após a roçada; DAAS: dias após a aplicação sequencial. (+) indicates that the treatment was superior to the standard and (-) indicates that the treatment was lower than the standard, by orthogonal contrast estimation, at 5\% probability. ${ }^{1 / h e r b i c i d e ~ o n l y ~(c h e t h o d i m ~}+$ glyphosate -192 and $1080 \mathrm{~g} \mathrm{ha}^{-1}$ ). All treatments received two sequential applications: application Seq.1 - 25 days after mowing with clethodim + glyphosate $\left(192+1080 \mathrm{~g} \mathrm{ha} \mathrm{a}^{-1}\right)$. Application Seq. 2 - 45 days after mowing with clethodim + glyphosate $\left(108+1080 \mathrm{~g} \mathrm{ha} \mathrm{g}^{-1}\right)$. DAR: days after mowing; DAAS: days after sequential application.

Quanto mais rente ao solo o corte das plantas de capim-amargoso, melhores são os resultados do sistema de manejo com controle químico associado à roçada. Em gramíneas, quanto mais folhas e colmos sobram após o corte, maior é a interceptação de luz para a realização da fotossíntese e maior a chance de emissão de novos perfilhos (MACEDO et al., 2017), o que pode acelerar o processo de rebrote. Quando as plantas são roçadas rente ao solo, grande quantidade das gemas e folhas é eliminada, o que proporciona supressão na intensidade do rebrote.

Embora seja considerada de baixo rendimento operacional, a roçada do capim-amargoso constitui uma importante ferramenta para ser integrada ao controle químico em áreas de pousio ou períodos de entressafra infestados com plantas perenizadas. Dentre as vantagens apresentam-se a melhoria na operação da semeadura mecanizada, redução na produção de sementes, esgotamento das reservas das plantas e prevenção de aparecimento de resistência a herbicidas. Mais estudos são necessários para avaliar a viabilidade da roçada aliada ao controle químico compondo programas de manejo desde a entressafra até o final do ciclo da cultura da soja. 


\section{CONCLUSÃO}

A aplicação de clethodim + glyphosate $\left(192+1080 \mathrm{~g} \mathrm{ha}^{-1}\right)$ imediatamente após a roçada aumenta os níveis de controle final em até $4,8 \%$. Quanto menor a altura de roçada, maior é o controle de capim-amargoso, quando associada com o controle químico.

A roçada $\leq 20 \mathrm{~cm}$ com duas aplicações sequenciais de clethodim + glyphosate (108 ou $\left.192+1080 \mathrm{~g} \mathrm{ha}^{-1}\right)$ e clethodim + glyphosate $\left(108+1080 \mathrm{~g} \mathrm{ha}^{-1}\right)$ quando o rebrote atinge 15 $\mathrm{cm}$ proporciona bons níveis de controle de capim-amargoso $(83-98 \%)$ podendo substituir o controle exclusivamente químico.

\section{AGRADECIMENTOS}

Agradecemos à Capes pela concessão de bolsas de estudo e suporte para submissão desse trabalho.

\section{REFERÊNCIAS BIBLIOGRÁFICAS}

ADEGAS, F. S.; GAZZIERO, D. L. P.; VOLL, E.; OSIPE, R. Diagnóstico da resistência de Digitaria insularis ao herbicida glyphosate no Sul do Brasil. In: CONGRESSO BRASILEIRO DA CIÊNCIA DAS PLANTAS DANINHAS, 27, 2010, Ribeirão Preto. Anais... Ribeirão Preto: SBCPD, 2010. p. 761-765.

CARVALHO, L. B.; CRUZ-HIPOLITO, H.; GONZÁlES-TORRALVA, F.; ALVES, P. L. C. A.; CHRISTOFFOLETI, P. J.; PRADO, R. Detection of sourgrass (Digitaria insularis) biotypes resistant to glyphosate in Brazil. Weed Science, Cambridge, v. 59, n. 2, p.171-176, 2011.

CORREIA, N. M.; ACRA, L. T.; BALIEIRO, G. Chemical control of different Digitaria insularis populations and management of a glyphosate-resistant population. Planta Daninha, Viçosa, MG, v. 33, n. 1, p.93-101, 2015.

DANTAS, G.; MESCHEDE, D. K.; SAMABATTI, V. C.; AZEVEDO, G.; VANZELLA, L.; DENADAI, J.; GAZZIERO, D. L. P. Roçada como ferramenta no controle do capimamargoso (Digitaria insularis) resistente ao glyphosate na cultura da soja. In: CONGRESSO BRASILEIRO DE SOJA, 7, 2015, Florianópolis. Resumos... Florianópolis: Embrapa, 2015. p.1-3.

GEMELLI, A.; OLIVEIRA JR., R. S.; CONSTANTIN, J.; BRAZ, G. B. P.; CAMPOS, T. M. J.; GHENO, E. A.; FRANCHINI, L. H. M. Estratégias para o controle de capim-amargoso (Digitaria insularis) resistente ao glyphosate na cultura milho safrinha. Revista Brasileira de Herbicidas, Londrina, v. 12, n. 2, p.162-170, 2013.

GEMELLI, A.; OLIVEIRA JR., R. S.; CONSTANTIN, J.; BRAZ, G. B. P.; JUMES, T. M. C.; OLIVEIRA NETO, A. M.; DAN, H. A.; BIFFE, D. F. Aspectos da biologia de Digitaria insularis resistente ao glifosato e implicações para o seu controle. Revista Brasileira de Herbicidas, Londrina, v. 11, n. 2, p.231-240, 2012. 
GILO, E. G.; MENDONÇA, C. G.; ESPÍRITO SANTO, T. L.; TEODORO, P. E. Alternatives for chemical management of sourgrass. Bioscience Journal, Uberlândia, v. 32, n. 4, p.881889, 2016.

LICORINI, L. R.; GANDOLFO, M. A.; SORACE, M. A.; OSIPE, R.; COSSA, C. A.; OSIPE, J. B. Identificação e controle de biótipos resistentes de Digitaria insularis (L.) Fedde ao glyphosate. Revista Brasileira de Herbicidas, Londrina, v. 14, n. 3, p.141-147, 2015.

LISBOA, B. B.; VARGAS, L. K.; SILVEIRA, A. O.; MARTINS, A. F.; SELBACH, A. Indicadores microbianos de qualidade do solo em diferentes sistemas de manejo. Revista Brasileira de Ciência do Solo, Viçosa, v. 36, n. 3, p.45-55, 2012.

LOPEZ OVEJERO, R. F.; TAKANO, H. K.; NICOLAI, M.; FERREIRA, A.; MELO, M. S. C.; CAVENAGHI, A. L.; CHRISTOFFOLETI, P. J.; OLIVEIRA JÚNIOR, R. S. Frequency and dispersal of Glyphosate-resistant sourgrass (Digitaria insularis) populations across Brazilian agricultural production areas. Weed Science, Cambridge, v. 65, n. 2, p.285-294, 2017.

LORENZI, H. Manual de identificação e controle de plantas daninhas: plantio direto e convencional. 7. ed. Nova Odessa: Instituto Plantarum, 2014. 379 p.

MACEDO, V. H. M.; CUNHA, A. M. Q.; CÂNDIDO, E. P.; DOMINGUES, F. N.; MELO, D. M.; RÊGO, A. C. Estrutura e produtividade de capim-tanzânia submetido a diferentes frequências de desfolhação. Ciência Animal Brasileira, Goiania, v. 18, n. e-383984, p.1-10, 2017.

MARTINS, J. F.; BARROSO, A. A. M.; ALVES, P. L. C. A. Effects of environmental factors on seed germination and emergence of glyphosate resistant and susceptible sourgrass. Planta Daninha, Viçosa, v. 35, n. e 017164499, p.1-8, 2017.

MElO, M. S. C.; ROSA, L. E.; BRUNHARO, C. A. C. G.; NICOLAI, M.; CHRISTOFFOLETI, P. J. Alternativas para o controle químico de capim-amargoso (Digitaria insularis) resistente ao glyphosate. Revista Brasileira de Herbicidas, Londrina, v. 11, n. 2, p.195-203, 2012.

MENDES, R. R.; BIFFE, D. F.; CONSTANTIN, J.; OLIVEIRA JÚNIOR, R. S.; ROSA, E. L.; CUBA, A. L. F.; BALADELI, R. B. Controle de amarelinho (Tecoma stans) em pastagem com aplicações localizadas de herbicidas. Revista Brasileira de Herbicidas, Londrina, v. 15, n. 4, p.303-312, 2016.

RAIMONDI, R. T. Alturas de roçada combinadas à aplicação de herbicidas visando ao controle de capim-amargoso (Digitaria insularis). 2018. 58 f. Dissertação (Mestrado em Agronomia) - Universidade Estadual de Maringá, Maringá, 2018.

SILVA, M. C. C.; BRAUN, H.; COELHO, F. S. Manejo e controle de plantas daninhas na cultura da batata. Revista Brasileira de Agropecuária Sustentável, Viçosa, v. 1, n. 1, p.6067, 2011.

SILVA, W. T.; KARAM, D.; VARGAS, L.; SILVA, A. F. Alternativas de controle químico para capim-amargoso (Digitaria insularis) na cultura do milho. Revista Brasileira de Milho e Sorgo, Sete Lagoas, v. 16, n. 3, p.578-586, 2017. 
SOCIEDADE BRASILEIRA DA CIÊNCIA DAS PLANTAS DANINHAS - SBCPD. Procedimentos para instalação, avaliação e análise de experimentos com herbicidas. Londrina - PR: S.B.C.P.D., 1995. 42 p.

ZOBIOLE, L. H. S.; KRENCHINSKI, F. H.; ALBRECHT, A. J. P.; PEREIRA, G.; LUCIO, F. R.; RUBIN, R. S. Controle de capim-amargoso perenizado em pleno florescimento. Revista Brasileira de Herbicidas, Londrina, v. 15, n. 2, p.157-164, 2016. 